\title{
Moving Robots Lies and Their Minds with Degree of Confidence in a Decentralized Autonomous FMS
}

\author{
Hidehiko Yamamoto \\ Department of Mechanical Engineering, Gifu Univ., 1-1, Yanagido \\ Gifu-shi, Gifu, 501-1193, Japan \\ Takayoshi Yamada \\ Department of Mechanical Engineering, Gifu Univ., 1-1, Yanagido \\ Gifu-shi, Gifu, 501-1993, Japan \\ Shizuka Tanaka \\ Mitsubishi Electric Mechatronics Software Corporation, Nagoya, Aichi, Japan \\ E-mail:yam-h@gifu-u.ac.jp,yamat@gifu-u.ac.jp \\ wwwl.gifu-u.ac.jp/ yamlab/
}

\begin{abstract}
According to the previous study of a decentralized autonomous FMS using a mind, it is found that the production outputs decreased because an agent sometimes lies. To solve this problem, we study an AGV's lie. The study is that AGVs recognize the lie that each AGV sends. To do this, we propose to give a degree of confidence to the mind that each AGV has. By performing the production simulations with the degree of confidence, we improve the increase of the production outputs.
\end{abstract}

Keywords: Decentralized Autonomous FMS, Lie, Mind, Degree of Confidence, AGV.

\section{Introduction}

We've developed the computer control systems to realize a decentralized autonomous flexible manufacturing system (FMS). To do this, it is necessary to avoid the path interference of automated guided vehicles (AGVs) in a decentralized autonomous FMS. To solve this problem, we inserted an arrogant mind and a modest mind into AGVs to control AGVs' moving.

In a decentralized autonomous FMS with minds, it is assumed that the information provided by the agents is always correct. However, this is not the case in a real factory, where incorrect information can sometimes be sent as a result of machine failures. In this study, we define and find this incorrect information as a "lie" of an $\mathrm{AGV}$. Then, in the decentralized autonomous FMS including AGVs lies, we perform the moving control of the AGVs in order not to have a low productivity.
Specifically, we propose the method of finding lies using the AGV's position information. We also propose the method of controlling the AGV's moving using a degree of confidence.

\section{Mechanism of Decentralized Autonomous FMS}

\subsection{Description of Decentralized Autonomous FMS}

Fig. 1 shows the example of a decentralized autonomous FMS factory. The factory floor has been divided into a grid pattern where AGV moves on the line. The system of the automated factory performs the production by carrying the product to parts of the warehouse and machining centers (MCs). A decentralized autonomous FMS does not have a management mechanism that controls the whole system and the agents corresponding to the elements of the FMS determine their behaviors by the knowledge exchanges. 


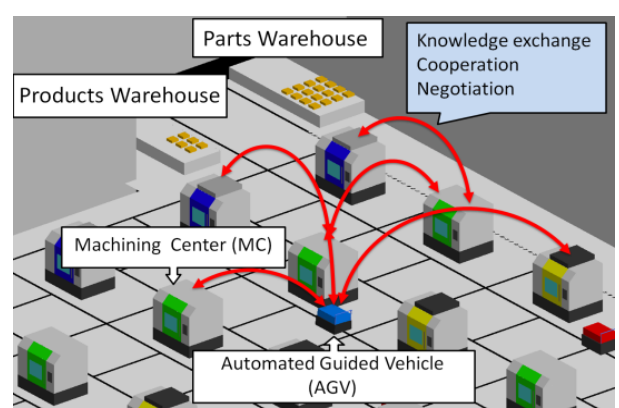

Fig.1. Example of decentralized autonomous FMS

\subsection{AGV', Behaviors and A Mind Model}

In the decentralized autonomous FMS, all AGVs use the position information received from the other AGVs and determine each behavior autonomously. In order to realize the autonomous behavior, we give a mind to each AGV. In the present study, in order to perform the behavior efficiently, we give an arrogant mind or a modest mind to AGVs. AGV with an arrogant mind takes action approaching the destination forcibly. AGV with a modest mind takes the action to make way to other AGV.

This mind is expressed in the mind model in Fig.2, Minimum Unit of Mind (MUM) ${ }^{[1]}$. A1 and A2 of MUM are called as unit, $\mathrm{X}$ is load, and the arrow is a stimulation vector. The threshold is determined by the unit. If the internal value reaches the threshold, we call it "excited". And if it does not reach the threshold, we call it "normal".

When a signal is sent to the unit, it sends a signal to the direction of the arrow if it is excited, and it is not sent if it is normal. Load has the function to change the internal value of the unit. When the signal is sent to the load, the value of the unit is increased by the value of $X$. Stimulation vector is a line connecting the load and the unit, and it gives a signal to the load or unit if the signal comes. When A1 is kept excited, we call it "Modest mind", and when normal, we call it "Arrogant mind".

By the amount of the signal input to the unit, MUM frequently changes the mind to an arrogant or a modest.

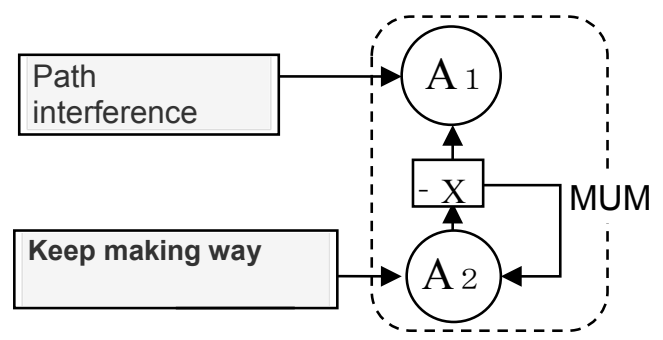

Fig.2. MUM
Next, we describe the internal functions of MUM. When the arrogant AGV has a path interference, A1 is increased by 1 . Keeping the situation of the interference and being increased by 1 , the A1 value becomes the threshold, and the AGV is changed to Modest mind.

When AGVs with Modest mind keep making way, the value of $A 2$ is increased by 1 . When the situation is repeated at optional times, A2 becomes excited and a signal is sent to a load. The received load decreases the values of units $\mathrm{A} 1$ and $\mathrm{A} 2$ by optional values. Owing to this, A1 and A2 are returned to normal and Modest mind is changed to Arrogant mind. In this way, AGV avoids the path interferences by the change of mind.

\section{How to Find AGV's Lies}

In the decentralized autonomous FMS using the mind, MUM, from our previous studies, each agent transmits the information, treats the received information as all correct and works in cooperation each other. However, breakdowns such as short-period stops occur in the real FMS factory. To realize this real factory in this study, we adopt the decentralized autonomous FMS where failure occurs. We define the AGV as "liar" when the AGV sends an incorrect information and "honest" when the AGV sends a correct information.

AGV's lies are defined as the following three types. 1. Although the current position is correctly taken, an AGV sends an incorrect information to other AGVs. We call this as "USO1".

2. An AGV sometimes takes the current position for a different one and sends it. We call this as "USO2".

3. An AGV sometimes fails to read the current position and does not update the information. We call this as "USO3".

The state of the USO1 is shown in Fig 3. When USO1 occurs, a correct position information is saved in the AGV that lied. However, an incorrect information is saved in the other AGVs.

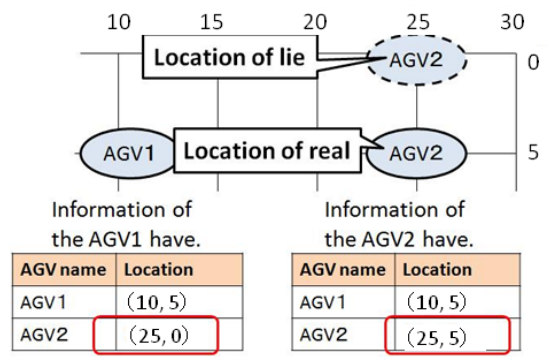

Fig.3. USO1 
The state of the USO2 is shown in Fig 4. When USO2 occurs, both the AGV that lied and other AGVs save an incorrect information.

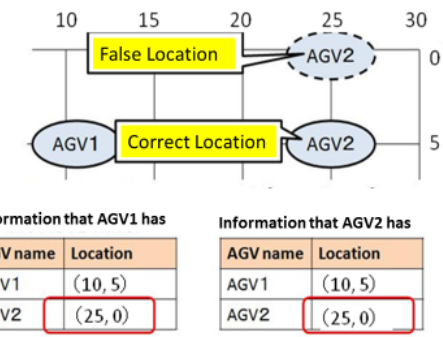

Fig.4. USO2

The state of the USO3 is shown in Fig 5. When USO3 occurs, the AGV that lied fails to read the current position. The AGV does not update the location information and does not send the information to other AGVs.

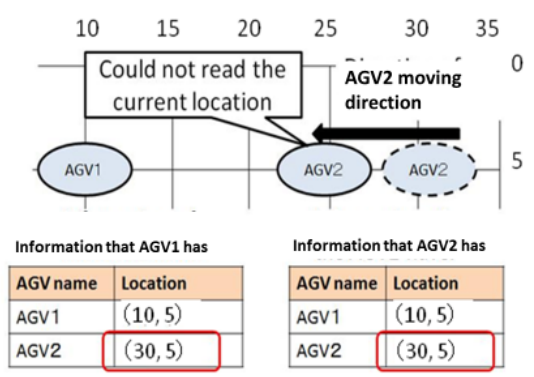

Fig.5. USO3

We find the three methods of the above lies.

[1] The AGVs except for the liar AGV receive the information from the liar AGV, compare the liar AGV's current position with its previous positions and find the incorrect information. This is the method to find USO1.

When the information transmitted from the other $\mathrm{AGV}$ satisfies Equation (1), the $\mathrm{AGV}$ that has transmitted the information is defined as USO1.

|(Past X-coordinate)-(Current X-coordinate)|

$+\mid($ Past Y-coordinate)-(Current Y-coordinate $) \mid>5$

[2] When the difference between the own current position and the previous position becomes big, the situation is called as USO2. When the AGV's information satisfies Equation (2), the AGV realizes as USO2.

|(Past X-coordinate)-(Current X-coordinate)|

$+\mid($ Past Y-coordinate)-(Current Y-coordinate) $\mid>10$ (2)

[3] This is the method to find the USO3. When the AGV's information satisfies Equation (3), the AGV realizes as USO3

|(Past X-coordinate)-(Current X-coordinate)|

$+\mid$ (Past Y-coordinate)-(Current Y-coordinate $) \mid=10$

\section{New Mind with Degree of Confidence}

If there are AGVs that lie, an $\mathrm{AGV}$ that uses the incorrect information takes the wrong action. As a result, the number of production is reduced. In this study, we adopt an honest AGV to move preferentially to solve this problem. To do this, we find the AGV that lie by distinguishing honest AGVs from liar AGVs. In order to achieve the distinguishing, we propose the new mind model that MUM includes the degree of confidence. Because of the new mind model, we can control that the higher confidence AGV preferentially moves.

In this study, we define the degree of confidence as "the degree which an AGV can correctly work without a lie". When an AGV passes through the intersection without a lie, the confidence of the mind of the AGV is increased. The higher the confidence, the more arrogant the AGV becomes. Because of this, the AGV does the work preferentially.

The way to give confidence is shown as the following.

1. When an $\mathrm{AGV}$ is passed through an intersection without a lie, the mind of confidence is increased by 1 .

2 . If the confidence reaches a threshold, the threshold value of the unit A1 is increased by 1 , and the mind sets the confidence as 0 .

The new mind model of Fig.6. is generated by adding MUM of Fig2 to a unit C, load Y, Z, and the stimulus vector. We call it as MUMS (MUM with Selfconfidence degree). Unit C of MUMS is called degree of confidence; unit $\mathrm{C}$ has a threshold $\mathrm{C}[\mathrm{T}]$ and excitement value $\mathrm{C}[\mathrm{E}]$. Load $\mathrm{Y}$ indicating by the triangle of Fig. 6 sends a signal to the threshold A1[T]. Load $-Z$ sends a signal to the excitement value $C[E]$ of the unit $\mathrm{C}$.

Further, the functions of the MUMS are shown below. When an AGV passes through the intersection

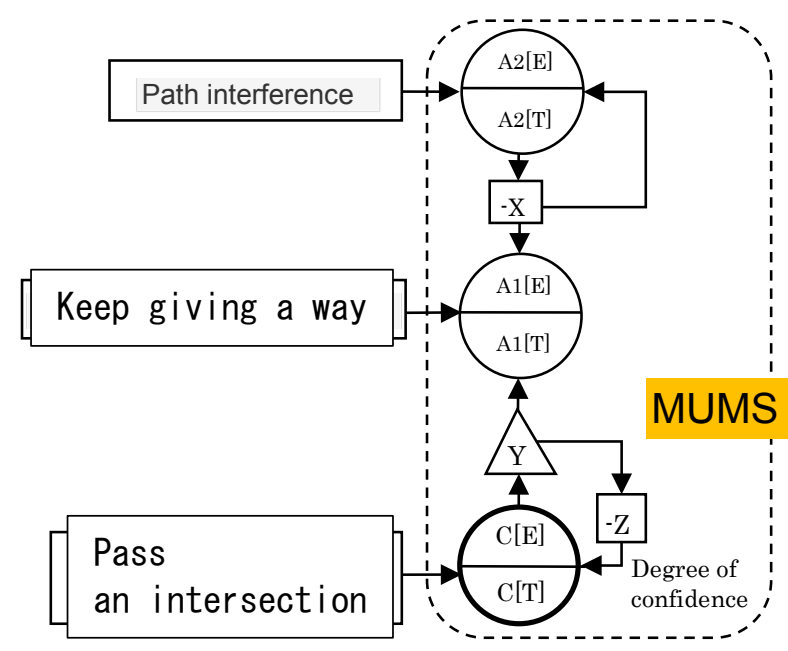

Fig.6 MUMS 
without a lie, a signal is sent to $C[E]$. In other words, the degree of confidence is increased. When $\mathrm{C}[\mathrm{E}]$ continues to receive the signal, the unit $\mathrm{C}$ becomes excited and sends a signal to the load $\mathrm{Y}$ and load $-\mathrm{Z}$. The load $\mathrm{Y}$ increases $A 1[T]$ and $C[E]$ is reduced by the load $-Z$. In this way, when an AGV passes through the intersection without a lie, A1[T] is increased. When A1 [T] increases, the unit A1 is hard to become the excited condition, that is, it is easy to have an arrogant mind. On the other hand, when an AGV passes through the intersection with a lie, the signal is not sent to the unit $\mathrm{C}$. Because of it, A1[T] is not changed.

\section{Simulation Results and Considerations}

We simulated the production results of FMS including MUMS. We set AGVs lie with a certain probability and carried out the production simulations by using the following four conditions.

- FMS just using honest AGVs

- FMS including AGVs that lie

- FMS including AGVs that lie, and the AGVs with MUMS (Set the threshold value C [T] shown in Fig.6 as 500)

- FMS including AGVs that lie, and the AGVs with MUMS

(Set the threshold value C [T] shown in Fig.6 as 900)

The production simulations were carried out seven times under these conditions. Table. 1 shows the average of the output of simulation production, the total number of collisions and the efficiency of each AGV. Table.2 also shows the adopted stimulus values of the degrees of confidence of AGVs.

From Table.1, comparing the honest FMS with the FMS including liar AGVs, the latter FMS reached to a

Table.1. Simulation Results

\begin{tabular}{|c|c|c|c|c|c|}
\hline & & $\begin{array}{c}\text { Honest } \\
\text { AGVs }\end{array}$ & $\begin{array}{c}\text { liar } \\
\text { AGVs }\end{array}$ & $\begin{array}{c}\text { Degree of } \\
\text { Confidence } \\
(500)\end{array}$ & $\begin{array}{c}\text { Degree of } \\
\text { Confidence } \\
(900)\end{array}$ \\
\hline \multicolumn{2}{|c|}{ Outputs } & 158.7 & 133.7 & 138.4 & 140.4 \\
\hline \multicolumn{2}{|c|}{$\begin{array}{l}\text { Collision } \\
\text { Number }\end{array}$} & 0 & 2159.9 & 2385.1 & 2236.7 \\
\hline \multirow{5}{*}{ 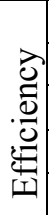 } & AGV1 & 0.591 & 0.414 & 0.408 & 0.424 \\
\hline & AGV2 & 0.901 & 0.716 & 0.707 & 0.718 \\
\hline & AGV3 & 0.847 & 0.646 & 0.622 & 0.642 \\
\hline & AGV4 & 0.686 & 0.506 & 0.495 & 0.507 \\
\hline & AGV5 & 0.774 & 0.559 & 0.551 & 0.569 \\
\hline
\end{tabular}

Table.2. Stimulus values of the degree of confidence

\begin{tabular}{|c|c|c|c|c|c|}
\hline & AGV1 & AGV2 & AGV3 & AGV4 & AGV5 \\
\hline $\mathrm{C}[\mathrm{T}]=500$ & 2 & 11 & 9 & 8 & 9 \\
\hline $\mathrm{C}[\mathrm{T}]=900$ & 0 & 6 & 4 & 6 & 4 \\
\hline
\end{tabular}

bad production results, 25 products lower than the former FMS. Comparing the latter FMS with the FMS including AGVs with MUMS, the number of production for the FMS with MUMS was increased. The number of production for each condition, 500 and 900, was increased. The number of production with MUMS was close to the number of production with the honest FMS. Because AGVs that have the characteristics easy to become arrogant and modest by the confidence, the time required path interference avoidances was reduced.

In addition, comparing 500 with 900 , the number of production for 900 is two more than 500. Table.2 shows that the case of $\mathrm{C}[\mathrm{T}]=500$ gave a lot of stimulations to the mind of $\mathrm{AGVs}$. If $\mathrm{C}[\mathrm{T}]$ is too small, many signals are sent to $\mathrm{A} 1[\mathrm{~T}]$ and $\mathrm{A} 1[\mathrm{~T}]$ becomes too large. The bigger $\mathrm{A} 1[\mathrm{~T}]$ is, the longer the time that the change of mind starts is. As a result, the time to perform the judgment of the path interference avoidance becomes longer. Because of this, the magnitude of $\mathrm{C}[\mathrm{T}]$ affects the ease of the change of mind and the number of production.

However, even using the degree of confidence, it was not possible to reduce the total interference number. This is because the interference occurs together with the lie. Even if using the degree of confidence, it is not possible to reduce the number of the lie itself.

\section{Conclusions}

This study found how to find the lie and to decide the degree of confidence useful for improving the production number in a decentralized autonomous FMS including AGVs that lie. This study also found that the magnitude of $\mathrm{C}[\mathrm{T}]$ affects the ease of the change of mind and the number of production. We can establish the foundation of the way how to handle the lie.

In this study, the threshold value of the degree of confidence was determined in accordance with a certain probability of the lie happening. In the actual FMS factory, however, the agents that lie are not only AGVs but Machining Centers. We will study MCs lies in the future to realize the real FMS.

\section{References}

1. Hidehiko YAMAMOTO, Takayoshi YAMADA and Shinsuke KATO, AGV Mind Model and its Usage for Decentralized Autonomous FMS by Change of Mind, Proceeding of Third KES International Symposium, KESAMSTA 2009, Agent and Multi- Agent Systems : Technologies and Applications, Lecture Notes in Artificial Intelligence, Edited by Anne Hakansson, Ngoc Thanh Nguyen etc. , ISSN 0302-9743, Uppsala, Sweden, pp.744753, June(2009), Springer. 